\title{
The effect of Cordyceps extract and a mixture of Ganoderma lucidum/Agaricus Blazi Murill extract on human endometrial cancer cell lines in vitro
}

\author{
JENS C. HAHNE*, SUSANNE R. MEYER ${ }^{*}$, JOHANNES DIETL and ARND HONIG \\ Department of Gynecology, Medical University of Würzburg, D-97080 Würzburg, Germany
}

Received December 20, 2013; Accepted February 19, 2014

DOI: $10.3892 /$ ijo.2014.2414

\begin{abstract}
Endometrial carcinoma is the most common gynaecological malignancy. Nevertheless there is a lack of curative therapies, especially for patients diagnosed with late stage, recurrent or aggressive disease, who have a poor prognosis. Cordyceps Sinensis, Ganoderma lucidum and Agaricus Blazi Murill are three fungi widely used in traditional Chinese medicine, and effects as adjuvants in tumour therapy have been demonstrated. However, the function and effects of these fungi in regard to endometrial cancer are not known. Three endometrial cancer cell lines, Ishikawa, Hec-1A and AN3-CA (derived from endometrial cancers grade I, II and III, respectively), were used to determine the effect of the fungi extracts on endometrial cancer cell function and to analyze the molecular mechanism. All fungi extracts had an inhibitory effect on cell viability and proliferation most probably exerted through induction of autophagy. Our data suggest that these fungi extracts may be used as adjuvants in endometrial tumour therapy.
\end{abstract}

\section{Introduction}

Endometrial cancer is the most common gynecological malignancy in developed countries and represents the eighth leading cause of cancer related death in women (1). Apart from surgery, irradiation, hormonal-therapy and chemotherapy are used to cure this malignancy (2). The majority of patients with advanced endometrial cancers relapse because a proportion of primary tumours are intrinsically refractory to treatment. Therefore, a definite need for new treatment strategies exists.

Cordyceps Sinensis (Cordy), Ganoderma lucidum (Reishi) and Agaricus Blazi Murill (ABM) are fungi widely used in

Correspondence to: Professor Arnd Honig, Department of Gynecology, Medical University of Würzburg, Josef-Schneider-Str. 4, D-97080 Würzburg, Germany

E-mail: arnd_hoenig@hotmail.com

*Contributed equally

Key words: Cordyceps Sinensis, Ganoderma lucidum, Agaricus Blazi Murill, endometrial cancer, autophagic cell death traditional Chinese medicine. The biological activities and numerous pharmacological effects such as antitumour, immunomodulatory, anti-inflammatory, anti-diabetes, antihepatitis, anti-hypercholesterolemia, anti-heart disease and anti-oxidant properties of Cordy, Reishi and $A B M$ have been well documented (3-5). Of course, every fungi contains a variety of bioactive compounds, including triterpenes, polysaccharides, sterols, nucleoside and nucleotides, as well as their degradation products and derivatives (6-8). Multiple compound-based drugs may provide important combination therapies that simultaneously influence multiple pharmacological targets and provide clinical efficacy beyond that of single compound-based drugs (9).

However, up to now the biological pathways involved in pharmacological activities of Cordy, Reishi and ABM are still not clear. Furthermore, no data exist on the effects of these fungi on endometrial carcinomas. Therefore, we studied the effects of a hot-water-extract derived from Cordy, Reishi and $A B M$ on endometrial cancer cells in vitro. We used endometrial cancer cell lines derived from different stages; e.g. Ishikawa cells (stage I tumour); Hec-1A cells (stage II tumour) and AN3-CA cells (stage III tumour). Our purpose was to examine the efficiency of crude extracts from Cordy alone and a mixture composed of Reishi and $A B M$ for treatment of endometrial cancer in vitro systems. After demonstrating growth-inhibition in a dose- and time-dependent manner, we started to enlighten the molecular basis for the observed inhibition of proliferation caused by the different fungi extracts.

\section{Materials and methods}

Cell culture. AN3-CA, Hec-1A and Ishikawa cells were obtained from American Type Culture Collection (Manassas, VA, USA). Hec-1A and Ishikawa cells were grown in RPMI1640 medium (PAA, Cölbe, Germany) supplemented with $10 \%$ fetal calf serum, $2 \mathrm{mM}$ glutamine, $1 \%$ penicillin/streptomycin and $0.5 \%$ sodium pyruvate solution. AN3-CA cells were cultured in Dulbecco's modified Eagle's medium (DMEM) (PAA) containing 10\% fetal calf serum, $2 \mathrm{mM}$ glutamine, $1 \%$ penicillin/streptomycin and $0.5 \%$ sodium pyruvate solution.

Fungi extracts. The Cordyceps Sinensis as well as the mixture composed of 50\% (w/w) Reishi and 50\% (w/w) ABM was supplied by MycoVital (Limeshain, Germany). Whole 
mushrooms were dried (below $35^{\circ} \mathrm{C}$ ) and homogenized to a powder by the manufacturer. The mushrooms were not treated with any compound or chemical. The mushroom extracts were directly ordered by the manufacturer and used within the indicated best-before date. A stock solution of $50 \mathrm{mg} / \mathrm{ml}$ was prepared as previously described (10). Briefly, the fungi powder was suspended by adding water and then boiled for $5 \mathrm{~min}$. After brief centrifugation, the supernatant was collected and sterile filtrated. The stock solution was stored at $4^{\circ} \mathrm{C}$ in the dark. Such a hot water extraction is used in most studies with mushrooms because some compounds such as polysaccharides are found inside indigestible cell walls and only hot water extraction can release and thereby maintaining the structural integrity of these compounds (11). Two different lot numbers were tested from both mushroom preparations. We observed no significant differences between the two different lot numbers.

Cytotoxicity MTT assay. To quantify the cytotoxicity of the fungi extracts the viability of cells was measured with a nonradioactive cell viability assay. Therefore, cells were cultured in 96-well flat-bottom plates, in humidified $37^{\circ} \mathrm{C}$ and $5 \% \mathrm{CO}_{2}$ atmosphere. The cell density was initially adjusted to $2 \times 10^{5}$ cells $/ \mathrm{ml}$ in a final volume of $50 \mu \mathrm{l} /$ well. Cells were treated with different concentrations of fungi extracts as indicated for 24,48 and $72 \mathrm{~h}$, respectively. During the last $4 \mathrm{~h}$ of incubation, cells were pulsed with $10 \mu \mathrm{l}$ of tetrazolium salt [3-(4,5-dimethylthiazol-2-yl)-2,5-diphenyltetrazolium bromide, MTT] labelling reagent (Roth, Karlsruhe, Germany) at a final concentration of $0.5 \mathrm{mg} / \mathrm{ml}$. The colorimetric assay is based on the reduction of yellow MTT to pure violet formazan crystals by metabolic active cells (12). The crystals were solubilised by addition of $100 \mu 110 \%$ SDS in $0.01 \mathrm{M} \mathrm{HCl}$ to each well. Absorbance was measured spectrophotometrically using a 540-nm wavelength ELISA reader (Tecan, Männedorf, Switzerland) and Magellan software. The experiments were performed 6-fold. At least 3 independent experiments were performed for each cell line.

Preparation of cell lysates and western blotting. Preparation of cell lysates was performed as previously described (13). Membranes were probed overnight with anti-PhosphoAKT antibody and anti-AKT-antibody from Epitomics (Burlingame, CA, USA), anti-LC3B antibody from Cell Signaling (Frankfurt, Germany) or anti- $\beta$-actin antibody from Abcam (Cambridge, UK), respectively. Secondary horseradish peroxidase (HRP)-conjugated antibodies were obtained from Cell Signaling. The chemiluminescent HRP substrate solution (Millipore, Schwalbach, Germany) was used for detection.

NK-cell preparation and lysis assay. PBMC were isolated from healthy volunteers by density gradient centrifugation (Biocoll; Biochrom AG, Berlin, Germany). Monocytes were depleted by adherence and the remaining non-adherent PBL were further cultured on irradiated (30 Gy) RPMI-8866 feeder cells to obtain polyclonal NK-cell populations (19). After 6 days of co-culture 500 units of IL-2 (Peprotech, Hamburg, Germany) were added per $\mathrm{ml}$ and after $48 \mathrm{~h}$ the polyclonal NK-cell population (effector cells) was used in different killing assays. Therefore the NK-cells were labeled with eFluor 670 (eBioscience, Frankfurt, Germany). The lytic activity against CFSE-stained (eBioscience) tumour cells (targets; $10^{5}$ cells/well) was assessed in a modified $5 \mathrm{~h}$ FATAL assay using various effector:target ratios (20). Cells were detached by trypsinisation. The target cell lysis was determined by flow cytometric analysis of 30,000 target cells in a FACScan flow cytometer (Calibur, BD Biosciences, Heidelberg, Germany). eFluor 670-negative target cells were selected by gating and the percentage of CFSE cells within this population was determined. Spontaneous leakage of CFSE was determined by incubating the target cells with medium alone.

Flow cytometry. For cell cycle analysis, cells were treated with fungi extracts as indicated, harvested, fixed and permeabilized overnight in ice-cold $70 \%$ ethanol (Merck, Darmstadt, Germany). The cells were washed twice with PBS. RNA was digested with RNase A (Gibco Life Technologies, Paisley, UK). The DNA was stained with propidium iodide $(50 \mu \mathrm{g} / \mathrm{ml})$. Fluorescence was recorded in a FACSCalibur (Becton Dickinson, Heidelberg, Germany). Instrument settings were adjusted to move the G0/G1 peak to 200 relative fluorescence units. Cells to the left of this peak appeared to have DNA content below 2n, indicative of cell death. Aggregated cells were gated out. A total of $2 \times 10^{4}$ cells per condition were recorded.

Apoptosis assay. Cellular apoptosis was measured by Annexin V and propidium iodide staining using Annexin V Apoptosis Detection kit FITC (eBioscience) according to the manufacturer's protocol. Briefly, cells were treated with fungi extracts as indicated, harvested, washed once with binding buffer [10 mM HEPES/NaOH (pH 7.4), $140 \mathrm{mM} \mathrm{NaCl,} 2.5 \mathrm{mM}$ $\left.\mathrm{CaCl}_{2}\right]$ and resuspended in binding buffer at a cell density of $1 \times 10^{6}$ cells $/ \mathrm{ml}$. FITC-conjugated Annexin V (5 $\left.\mu \mathrm{l}\right)$ was added to $100 \mu \mathrm{l}$ of the cell suspension and incubated $15 \mathrm{~min}$ at room temperature. Then the cells were washed with binding buffer and finally resuspended in $200 \mu \mathrm{l}$ binding buffer. After addition of $5 \mu \mathrm{l}$ propidium iodide $(2 \mu \mathrm{g} / \mathrm{ml})$ the cells were analyzed by flow cytometry on a FACSCalibur (Becton Dickinson).

Autophagy assay. The degree of autophagic death was measured by acridine orange (Sigma-Aldrich, St. Louis, MO, USA) staining as previously described (14). Briefly, cells were treated with fungi extracts as indicated and acridine orange was added at a final concentration of $1 \mathrm{mg} / \mathrm{ml} 20 \mathrm{~min}$ before the cells were harvested. Cells were removed from the plate with trypsin-EDTA, and collected in phenol red-free growth medium. A total of $1 \times 10^{4}$ cells per condition were analyzed by flow cytometry on a FACSCalibur (Becton Dickinson).

\section{Results}

First of all the effects of the different fungi extracts on the viability and growing of endometrial cancer cell lines were evaluated (Figs. 1 and 2). It is obvious that the tumour cells are killed by the ABM/Reishi (Fig. 1) and Cordyceps (Fig. 2) extracts in a time- and dose-dependent manner. The viability of all endometrial cancer cells are $<20 \%$ compared to untreated control cells after incubation with $10 \mathrm{mg} / \mathrm{ml}$ of ABM/Reishi extracts for $72 \mathrm{~h}$ (Fig. 1). Of all endometrial cell lines used in this study, AN3-CA seems to be the most sensitive cells 

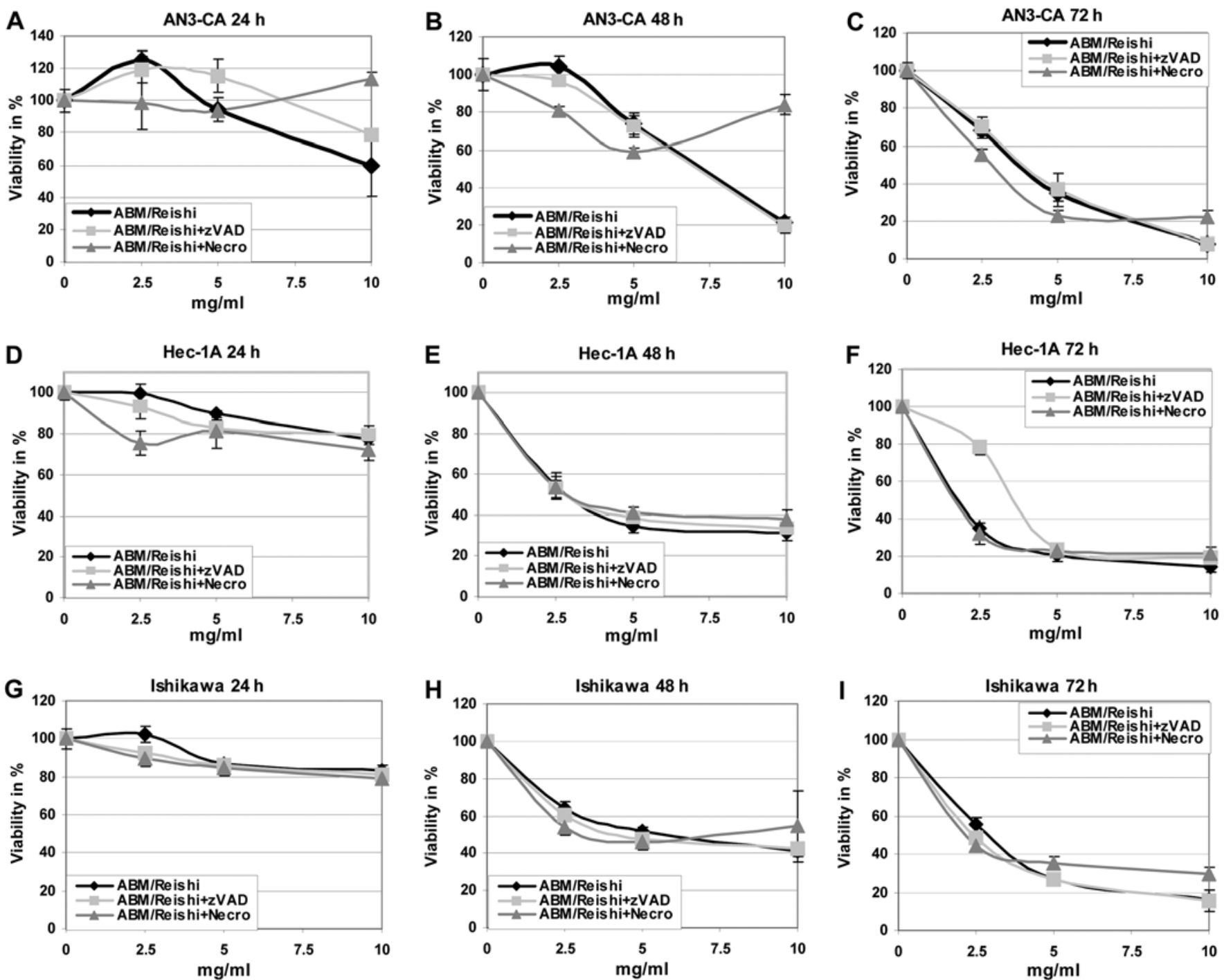

Figure 1. Growth inhibition mediated by ABM/Reishi extract in AN3-CA (A-C), Hec-1A (D-F) and Ishikawa (G-I) endometrial cancer cells. These human cancer cells were incubated for $24 \mathrm{~h}(\mathrm{~A}, \mathrm{D}$ and $\mathrm{G}), 48 \mathrm{~h}(\mathrm{~B}, \mathrm{E}$ and $\mathrm{H})$ and $72 \mathrm{~h}(\mathrm{C}, \mathrm{F}$ and $\mathrm{I})$ with increasing concentrations of $A B M / R e i s h i$ extract alone or in combination with the multi-caspase inhibitor z-VAD and necrostatin, respectively. The mean value of three independent experiments is shown.

for treatment with $A B M /$ Reishi extracts. Incubation for $48 \mathrm{~h}$ of AN3-CA cells with $10 \mathrm{mg} / \mathrm{ml}$ of $A B M /$ Reishi extracts resulted in killing of $80 \%$ of the cells (Fig. 1). Moreover, the incubation of endometrial cancer cells with Cordyceps extract resulted in a clearly reduced viability (Fig. 2). Only in Ishikawa cells the killing potency of Cordyceps extract is comparable to the effects observed with $A B M /$ Reishi extracts. In both other cell lines, the killing efficiency of Cordyceps extract is slightly diminished compared to the effects exerted by $A B M / R e i s h i$ extracts.

To further analyze the basis of the observed cytotoxic effects, cells were co-incubated with the fungi extracts and z-VAD-fmk, a broad caspase-inhibitor, or necrostatin-1, a necroptosis inhibitor, respectively (Figs. 1 and 2). Addition of z-VAD-fmk resulted in increased cell viability in AN3-CA cells after $24 \mathrm{~h}$ and in Hec-1A cells after $72 \mathrm{~h}$ of co-incubation with $A B M /$ Reishi extracts (Fig. 1A and F). Co-incubation of necrostatin with $A B M /$ Reishi extracts resulted in a significant protective effect only in AN3-CA cells at the highest concentration of $A B M /$ Reishi extracts used in this study at all time points as well as in Ishikawa cells after $72 \mathrm{~h}$ (Fig. 1A-C and I). In contrast, the co-incubation of Cordyceps extract with z-VAD-fmk or necrostatin-1 had only slight effects on the cell viability (Fig. 2).

In the next step, we addressed the question if the PI3K/ AKT pathway known to be highly active in a broad variety of tumours is influenced by the different fungi extracts. Because of the fact that phosphorylated AKT (pAKT) is indicative for the activity of the PI3K/AKT pathway, the expression level of pAKT as well as AKT was analyzed with specific antibodies in a western blot (Fig. 3). After incubation of AN3-CA cells with ABM/Reishi (Fig. 3A) or Cordyceps extracts (Fig. 3B) the pAKT expression was reduced. Prolonged incubation with fungi extracts resulted in a stronger inhibition of pAKT expression. In the same range, the total AKT expression is strongly reduced in AN3-CA cells by incubation with ABM/Reishi (Fig. 3A) and Cordyceps extracts (Fig. 3B). As expected in Hec-1A cells the pAKT expression is below the detection level, because of the wild-type PTEN expression in this cell line $(15,16)$. But nevertheless, the AKT expression is 



Figure 2. Growth inhibition mediated by Cordyceps extract in AN3-CA (A-C), Hec-1A (D-F) and Ishikawa (G-I) endometrial cancer cells. These human cancer cells were incubated for $24 \mathrm{~h}(\mathrm{~A}, \mathrm{D}$ and $\mathrm{G}), 48 \mathrm{~h}(\mathrm{~B}, \mathrm{E}$ and $\mathrm{H})$ and $72 \mathrm{~h}(\mathrm{C}, \mathrm{F}$ and $\mathrm{I})$ with increasing concentrations of Cordyceps extract alone or in combination with the multi-caspase inhibitor z-VAD and necrostatin, respectively. The mean value of three independent experiments is shown.
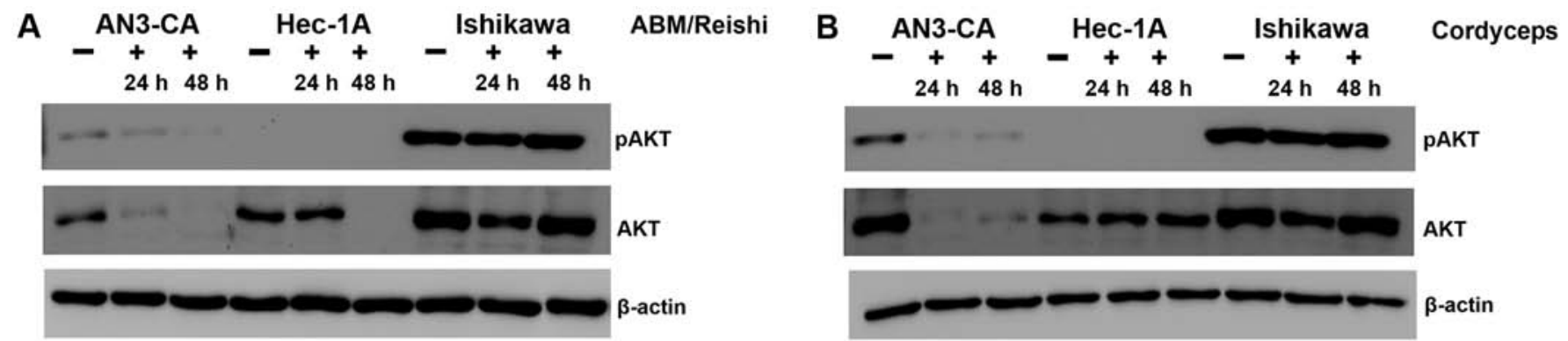

Figure 3. Western blotting showing expression of phosphorylated AKT-1 (pAKT) protein and the total amount of AKT-1 (AKT) in AN3-CA, Hec-1A and Ishikawa cells treated with (A) ABM/Reishi and (B) Cordyceps for 24 and $48 \mathrm{~h}$. In each lane $20 \mu \mathrm{g}$ of total protein was analysed by $10 \%$ SDS-polyacrylamide gel electrophoresis and transferred onto nitrocellulose. Membranes were probed overnight with anti-phospho-Akt antibody and anti-Akt antibody (Epitomics, Burlingame, CA, USA), respectively. A horseradish peroxidase-conjugated antibody was used as secondary antibody (Cell Signaling, Frankfurt, Germany). Antibodies were detected using chemiluminescent HRP substrate solution (Millipore, Schwalbach, Germany). The housekeeping protein $\beta$-actin was used as internal control.

strongly decreased by incubation with $A B M /$ Reishi extract for $48 \mathrm{~h}$ (Fig. 3A). In contrast, the incubation of Hec-1A cells with Cordyceps extract did not significantly alter the
AKT expression (Fig. 3B). In Ishikawa cells activated AKT (pAKT) was highly expressed due to an inactivating mutation of PTEN $(15,16)$ and only a slight decrease in the amount of 

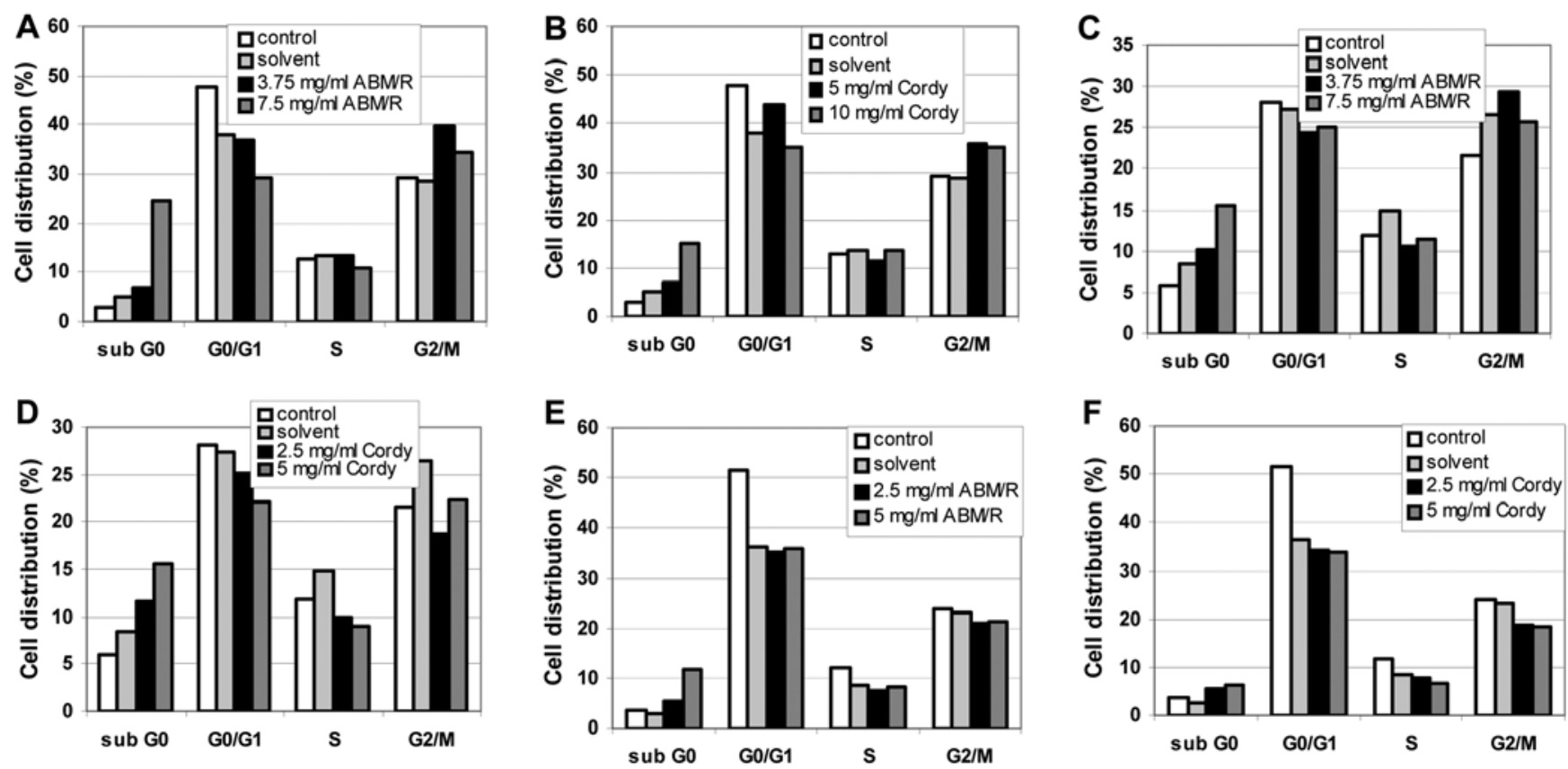

Figure 4. Effect of ABM/Reishi (ABM/R) extract (A, C and E) and Cordyceps (Cordy) extract (B, D and F) on the cell cycle distribution of human endometrial cancer cells. AN3-CA (A and B), Hec-1A (C and D) and Ishikawa (E and F) cells were treated with the indicated concentrations of the fungi extracts or solvent for $24 \mathrm{~h}$, fixed, permeabilized, stained with PI and analyzed by flow cytometry. The figure shows the distribution of the cells to the different phases of the cell cycle $(\%)$.
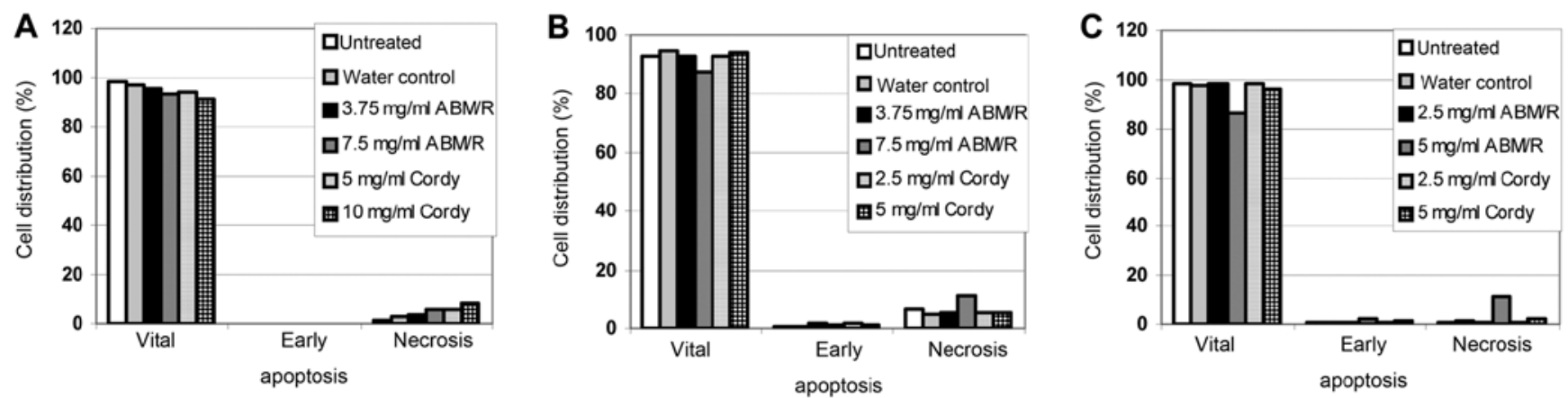

Figure 5. Measurement of cellular apoptosis and necrosis caused by ABM/Reishi (ABM/R) extract and Cordyceps (Cordy) extract in human endometrial cancer cells. AN3-CA (A), Hec-1A (B) and Ishikawa (C) cells were treated with the indicated concentrations of the fungi extracts or solvent for $24 \mathrm{~h}$ and stained with anti-Annexin V antibody (eBioscience, Frankfurt, Germany) and PI prior to flow cytometry measurement. The figure shows the amount of vital cells, early apoptotic cells and necrotic cells (\%).

pAKT was observed after incubation of these cells with the fungi extracts for $24 \mathrm{~h}$ (Fig. 3). Even in Ishikawa cells, the expression of AKT was reduced by both fungi extracts after incubation for $24 \mathrm{~h}$ (Fig. 3). Therefore, it seems that both fungi extracts exert at least to a certain degree their effect on the molecular level by decreasing AKT expression.

To enlighten further the effects of the fungi extracts on the endometrial cell lines, FACS analyses were performed. In cell cycle FACS analyses, an increase in the sub-G0 phase was observed after incubation of the cells (AN3-CA in Fig. 4A and B; Hec-1A in Fig. 4C and D; Ishikawa in Fig. 4E and F) for $24 \mathrm{~h}$ with the indicated concentrations of fungi extracts $[A B M /$ Reishi extract in Fig. 4A, C and E; Cordyceps (Cordy) extract in Fig. 4B, D and F] in all cases.

In FACS-based apoptosis assays, only slight effects were found after incubation of the cells (AN3-CA in Fig. 5A; Hec-1A in Fig. 5B; Ishikawa in Fig. 5C) for $24 \mathrm{~h}$ with the indi- cated concentrations of fungi extracts (Fig. 5). In agreement with the observation from the MTT assay with and without z-VAD-fmk (Figs. 1 and 2) no apoptotic cells were detected (Fig. 5). Furthermore, only a minor increase in necrosis was observed (Fig. 5). Therefore, it must be concluded that neither necrosis nor the programmed cell death (apoptosis) played a predominant role in the fungi extracts mediated cell death of endometrial cancer cells.

Another mechanism of programmed cell death often observed in tumour cells is autophagic death (17-19). To test the degree of autophagic death caused by both fungi extracts, the endometrial cells were stained with acridine orange after preincubation of the cells with Cordy and $A B M /$ Reishi extracts and thereafter analysed by FACS (AN3-CA in Fig. 6A; Hec-1A in Fig. 6B; Ishikawa in Fig. 6C). In all probes, incubation with fungi extracts for $24 \mathrm{~h}$ resulted in an increase in autophagy in comparison to the untreated control cells and the solvent 

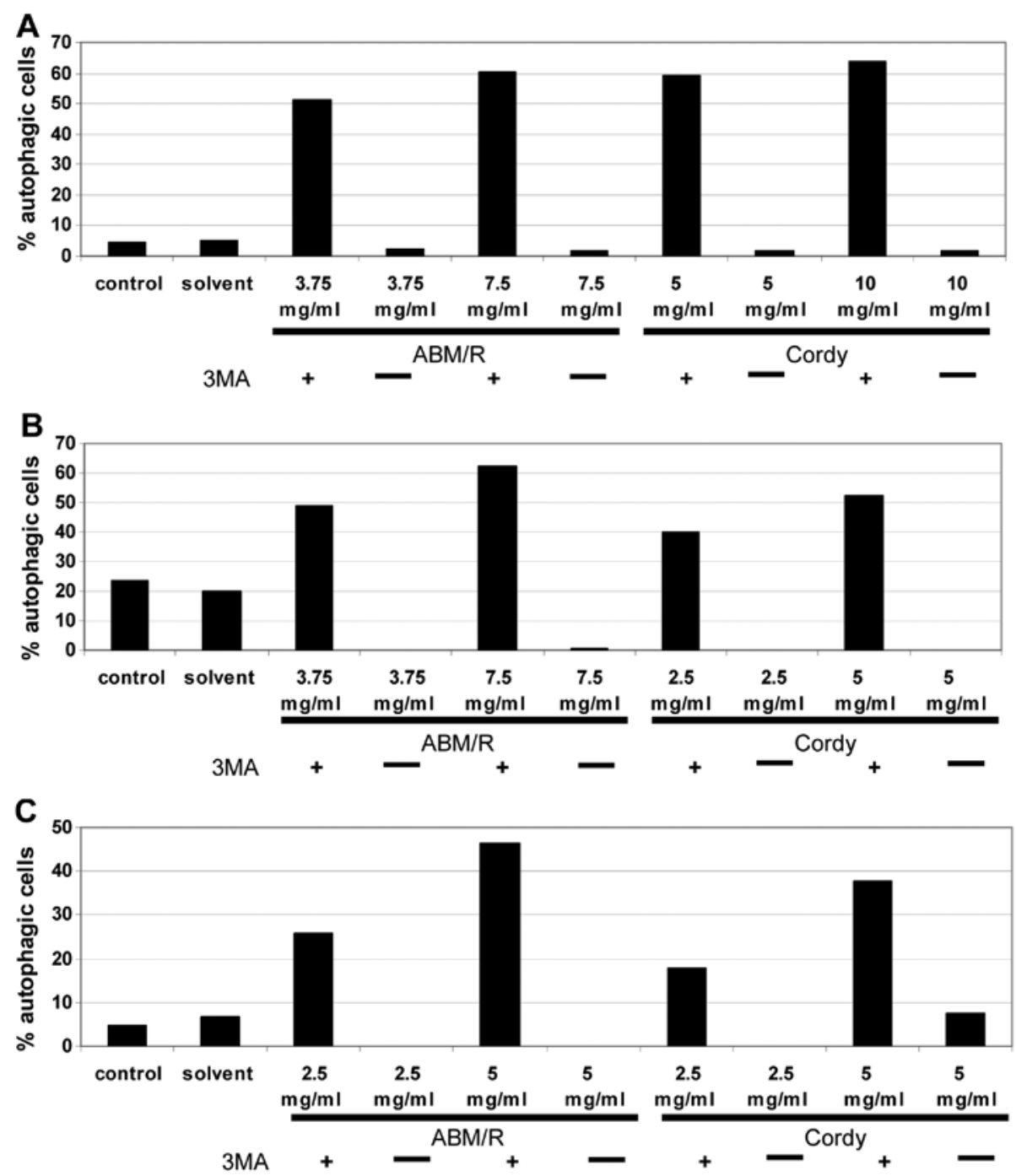

Figure 6. Measurement of autophagy caused by ABM/Reishi (ABM/R) extract and Cordyceps (Cordy) extract in human endometrial cancer cells. AN3-CA (A), Hec-1A (B) and Ishikawa (C) cells were treated with the indicated concentrations of the fungi extracts or solvent for $24 \mathrm{~h}$ and stained with acridine orange (Sigma-Aldrich, St. Louis, MO, USA) prior to flow cytometry analysis. By addition of the autophagy inhibitor 3'-methyladenine (3MA) the specificity was demonstrated. The figure shows the amount of autophagic cells (\%).

controls. To show the specificity the cells were co-incubated with the fungi extracts and the well established autophagy inhibitor 3'-methyladenine (3MA) (20) (Fig. 6). Surprisingly Hec-1A cells contained a high-level of autophagic cells even in the untreated control culture (Fig. 6B). In comparison to the control cultures of both other endometrial cancer cell lines in Hec-1A cells, nearly four times more autophagic cells were detected. By addition of 3'-methyladenine, the effects of the Cordy and ABM/Reishi extracts were completely abolished. Furthermore, the induction of autophagic death in endometrial cancer cells by Cordy and ABM/Reishi extracts were verified on the protein level by western blotting using a specific antibody against LC3 protein (Fig. 7). The conversion of LC3B-I to the faster migrating form LC3B-II is a well established indicator of autophagy. As shown for AN3-CA cells (Fig. 7A) and Ishikawa cells (Fig. 7C) incubation with fungi extract resulted in an increased amount of LC3B-II. In Hec-1A cells (Fig. 7B) it is difficult to detect an increase in LC3B-II after incubation with fungi extracts due to the already increased amount of autophagic cells in the untreated culture that was already observed in the FACS based detection of autophagy (Fig. 6B).

Since different fungi extracts are able to stimulate the non-specific immune system and to exert antitumour activity through the stimulation of the host's defence mechanism, we analyzed the effect on killing of endometrial cancer cells (AN3-CA in Fig. 8A; Hec-1A in Fig. 8B; Ishikawa in Fig. 8C) by NK-cells matured with and without addition of Cordy and ABM/Reishi extracts in the indicated concentrations (Fig. 8). The killing capacity of NK-cells was not significantly influenced by the maturation in the presence of either Cordy or ABM/Reishi extracts compared to NK-cells matured without addition of fungi extracts (Fig. 8). Furthermore, the incubation of tumour cells with the fungi extracts did not alter the killing efficiency of NK-cells (data not shown). Therefore, it seems that the used extracts from Cordy and ABM/Reishi do not influence the immunological interactions between tumour cells and NK-cells.

For treatment of endometrial cancer chemotherapeutics are often used, among those cisplatin is known to be one of the 
A
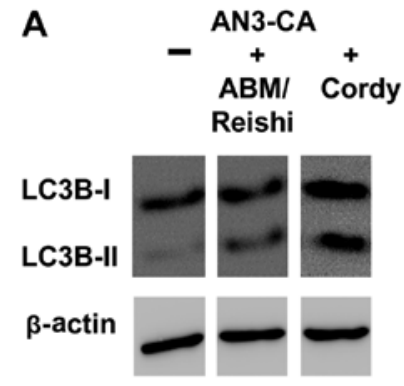

B
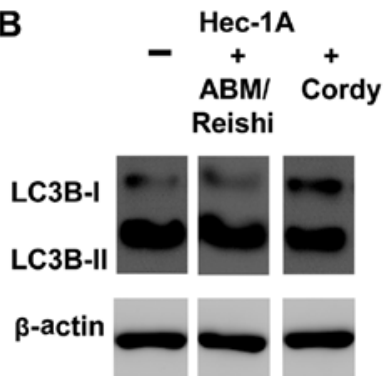

C
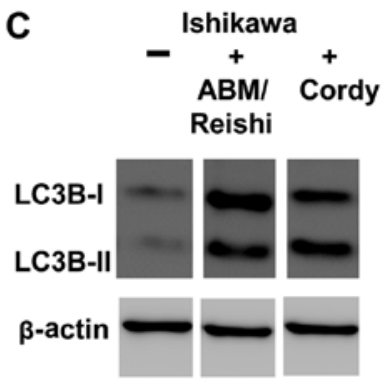

Figure 7. Western blotting showing expression of LC3B in AN3-CA (A), Hec-1A (B) and Ishikawa (C) cells. In each lane, $20 \mu \mathrm{g}$ of total protein was analysed by $10 \%$ SDS-polyacrylamide gel electrophoresis and transferred onto nitrocellulose. Membranes were probed overnight with anti-LC3B antibody, followed by incubation with a horseradish peroxidase-conjugated secondary antibody (both from Cell Signaling, Frankfurt, Germany). Antibodies were detected using chemiluminescent HRP substrate solution (Millipore, Schwalbach, Germany). The housekeeping protein $\beta$-actin was used as the internal control.
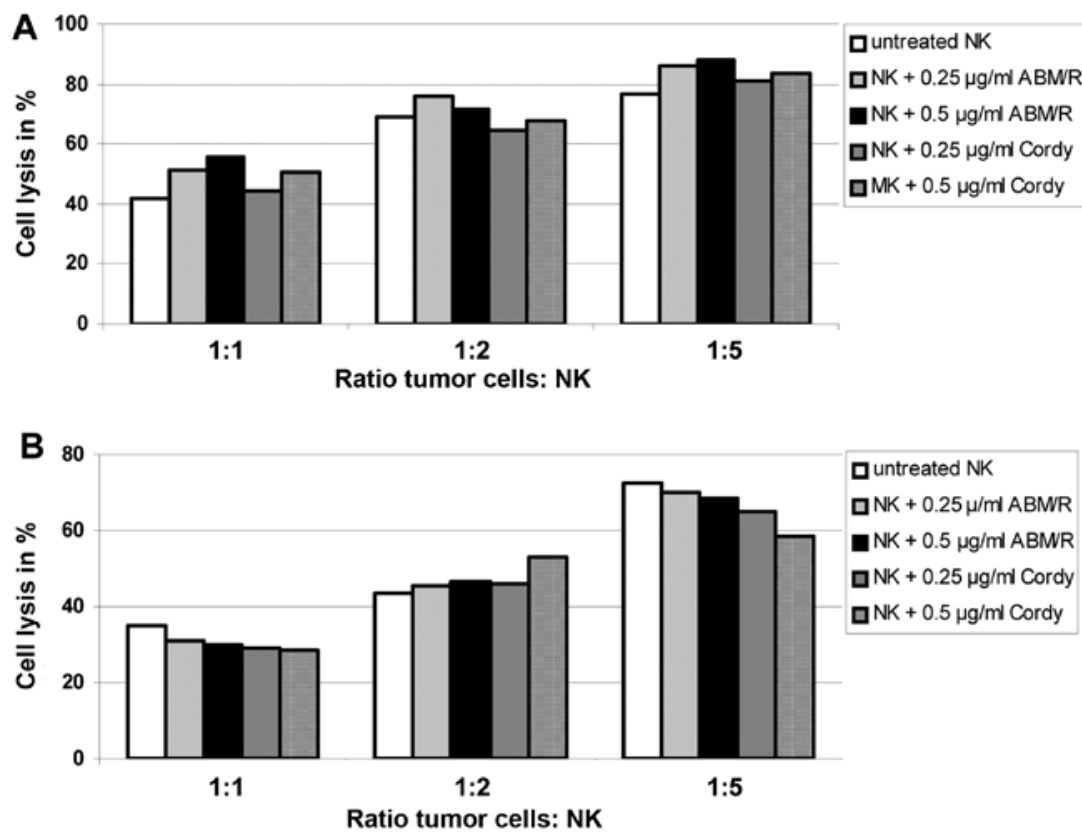

Ratio tumor cells: NK
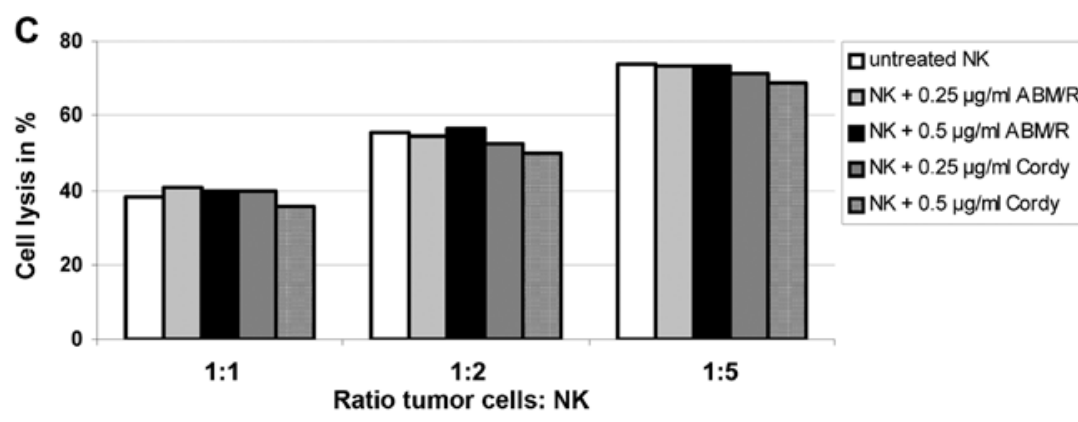

Ratio tumor cells: NK

Figure 8. Lytic activity of polyclonal natural-killer (NK) cells. AN3-CA (A), Hec-1A (B) and Ishikawa (C) cells (10 cells/well), respectively, were used as targets in a modified $5 \mathrm{~h}$ FATAL assay using various tumour cell:NK-cell ratios. Target cell lysis was determined by flow cytometric analysis. The percentage of tumour cell lysis was determined in relation to a control containing tumour cells with medium.

most effective in this disease. Therefore, we tested if combined effects can be observed by incubation of endometrial cancer cells with cisplatin and fungi extracts in comparison to treatment of the tumour cells with one of these substances alone. Exemplarily the results after incubation of the cells for $72 \mathrm{~h}$ are shown (Fig. 9). The incubation of the different endometrial cell lines with cisplatin in combination with ABM/Reishi (Fig. 9A-C) or Cordyceps (Fig. 9D-F) extracts resulted in a decreased viability compared to cisplatin incubation alone.

\section{Discussion}

Our purpose was to prove the efficiency of crude extracts from Cordy alone and a mixture composed of Reishi and $A B M$ for treatment of endometrial cancer in model systems in vitro. We have demonstrated here that hot-water-extract derived from Cordy and ABM/Reishi is able to suppress the growth of different human endometrial cancer cell lines in vitro. After this proof-of-concept the molecular basis was enlightened; 

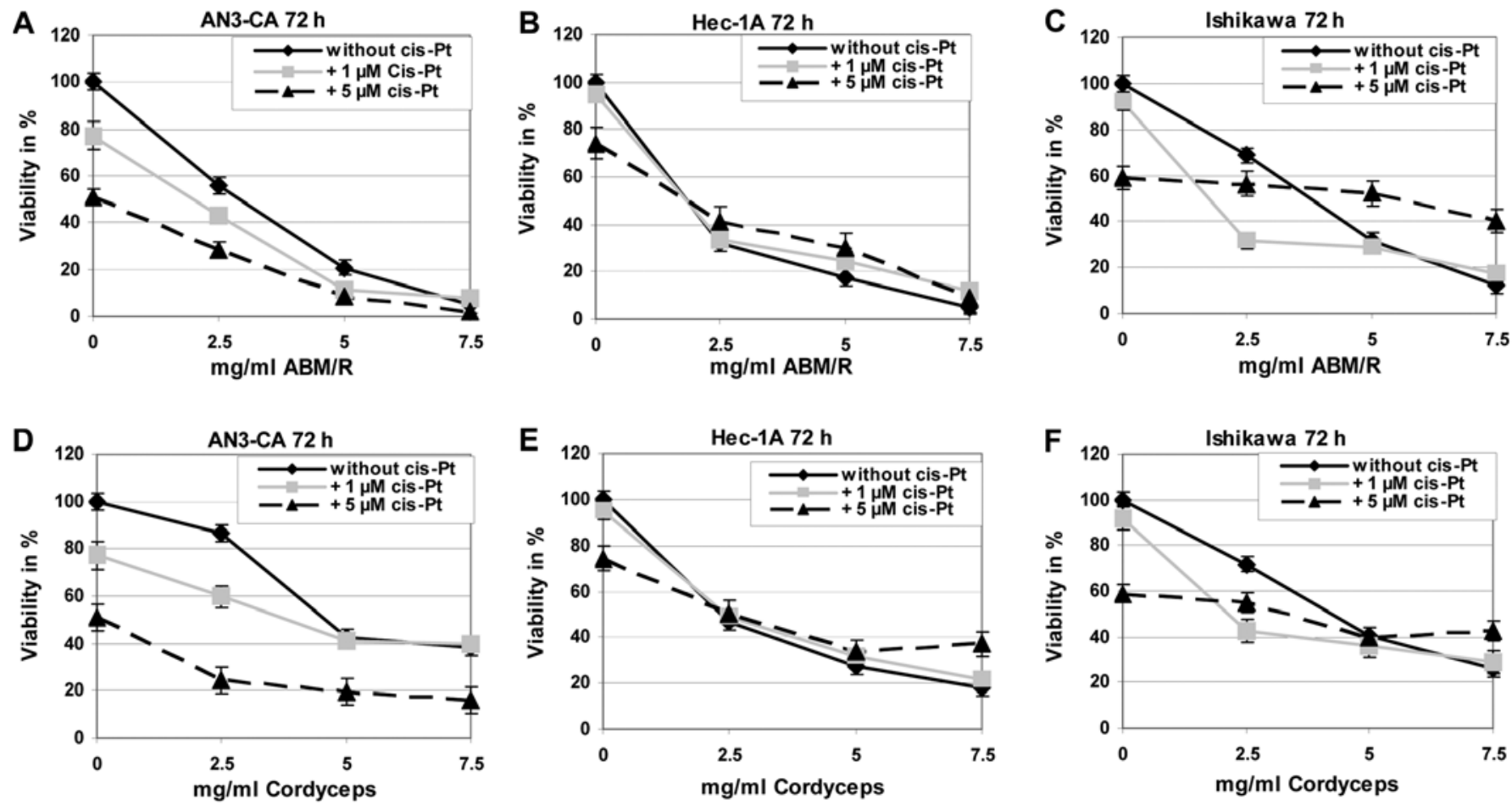

Figure 9. Growth inhibition mediated by ABM/Reishi (ABM/R) extract (A-C) and Cordyceps extract (D-F) in AN3-CA (A and D), Hec-1A (B and E) and Ishikawa $(\mathrm{C}$ and $\mathrm{F})$ endometrial cancer cells in combination with cisplatin. These human cancer cells were incubated for $72 \mathrm{~h}$ with increasing concentrations of $A B M /$ Reishi extract alone or in combination with 1 or $5 \mu \mathrm{M}$ cisplatin (cis-Pt), respectively. The mean value of three independent experiments is shown.

most probably the suppression of pAKT is involved in the observed antitumour effects. This finding in endometrial cancer cell lines is in agreement with previous studies on the effect of Cordy and Reishi extracts in leukemia cells as well as in ovarian, breast, prostate and gastric cancer $(10,21-25)$. The effective concentrations of fungi extracts in endometrial cancer cells are in the same range as previously described for ovarian cancer cells (10). Furthermore, our data argue for an interaction of the different fungi extracts with the PI3K/AKT signalling pathway known to be involved in a broad variety of cancers (26). We and others have examined the role of PI3K/AKT pathway in human tumours and we have recently demonstrated the role of pAKT level for different aspects of cancer cells, e.g. cisplatin based resistance and NK-cell mediated killing $(13,27)$. Moreover, it has been demonstrated that inhibition of the PI3K/AKT signalling pathway results in an increased tumour cell death (28-30). Another role of AKT has been published recently; it was demonstrated that in a panel of various human tumour types pAKT is necessary for phosphorylation of Beclin-1, an essential autophagy and tumour suppressor protein (31). If Beclin-1 is not phosphorylated increased autophagy, reduced anchorage-independent growth, and inhibited AKT-driven tumourigenesis was observed (31). In light of this observation, the correlation between increased autophagic death and decreased pAKT level in the endometrial tumour cells is explainable. In addition, the high amount of autophagic cells in the untreated cultures of Hec-1A cells (Fig. 6B) and the high expression level of LC3B-II (Fig. 7B) in these cells is most probably based on the low pAKT level in Hec-1A cells (Fig. 3).
Surprisingly the Cordy and ABM/Reishi extracts used in this study did not influence the interaction between endometrial tumour cells and NK-cells in experiments in vitro. Previously it has been reported that some polysaccharides or polysaccharide-protein complexes from mushrooms are able to stimulate the non-specific immune system and to exert antitumour activity through the stimulation of the host's defence mechanism (11,32-34). The fungi extracts activate effector cells such as macrophages, $\mathrm{T}$ lymphocytes and NK-cells to secrete cytokines (TNF- $\alpha$, IFN- $\gamma$ and IL-1 $\beta$ ), which are antiproliferative and induce apoptosis in tumour cells (11,32-34). All these effects of fungi extracts on the cells of the immune system have been evaluated in animal models and in human clinical practice (35) and clearly this complex interacting system of different stimulating and each other influencing cells that exist in vivo cannot be completely simulated in vitro. Therefore, our results concerning the tumour cell killing by NK-cells in an in vitro model must be re-analysed in an immunocompetent in vitro mouse model. But nevertheless our preliminary data show that no inhibitory effects are exerted by the different fungi extracts onto the NK-cell mediated tumour cell killing.

In any case, it should kept in mind that the origin as well as preparation of the fungi extracts can influence the effects to a very high degree as it was demonstrated exemplarily for breast cancer cells by Xie et al (36). Therefore, it is of pivotal importance in studies dealing with the effects of natural compound extracts to use one and the same standardized extraction method for the biological material from the same origin as we have done here and in previous studies (37). 
Because of the fact that every fungi contains various bioactive compounds, including triterpene, polysaccharides, sterols, nucleoside and nucleotides as well as their degradation products and derivatives (6-8) it is necessary to separate, isolate and analyze the different bioactive compounds in further studies. Nevertheless, multiple compound-based drugs may provide important combination therapies that simultaneously influence multiple pharmacological targets and provide clinical efficacy beyond that of single compound-based drugs (9).

In conclusion, to our knowledge this is the first study showing that fungi widely used in traditional Chinese medicine are able to suppress the growth of different human endometrial cancer cell lines in vitro. It seems to be very likely that the different fungi extracts act by suppression of AKT phosphorylation. The decreased level of pAKT results in an increased cell death. According to our data presented here an autophagic cell death is triggered by the fungi extracts in endometrial cancer cell lines. However, we must keep in mind that autophagy could act in two ways. Autophagy can play either pro-survival or pro-death roles $(17,38)$. Activation of autophagy may function as tumour suppressor mechanism by degrading cells or this pathway may be exploited by cancer cells to generate nutrients during periods of starvation and hypoxia $(17,19,39)$. Therefore, the Cordy and ABM/Reishi extracts may be used as adjuvants in endometrial tumour therapy in combination with chemotherapeutics to prevent tumour cells using the induced autophagy in a pro-survival manner.

\section{Acknowledgements}

We appreciate the permission to use the INTAS ChemoStar Imager (Department of Microbiology, University of Würzburg). Therefore, we thank especially Professor T. Rudel and Dr B. Bergmann. This work was supported by IZKF Würzburg.

\section{References}

1. Chaudhry P and Asselin E: Resistance to chemotherapy and hormone therapy in endometrial cancer. Endocr Relat Cancer 16: 363-380, 2009

2. Marnitz S and Köhler C: Current therapy of patients with endometrial carcinoma. A critical review. Strahlenther Onkol 188: $12-20,2012$.

3. Patel S and Goyal A: Recent developments in mushrooms as anticancer therapeutics: a review. 3 Biotech 2: 1-15, 2012.

4. Lindequist U, Niedermeyer TH and Jülich WD: The pharmacological potential of mushrooms. Evid Based Complement Alternat Med 2: 285-299, 2005.

5. Lima CU, Cordova CO, Nóbrega Ode T, Funghetto SE and Karnikowski MG: Does the Agaricus blazei Murill mushroom have properties that affect the immune system? An integrative review. J Med Food 14: 2-8, 2011.

6. Zhu JS, Halpern GM and Jones K: The scientific rediscovery of a precious ancient Chinese herbal regimen: Cordyceps sinensis: part II. J Altern Complement Med 4: 429-457, 1998.

7. Li SP, Yang FQ and Tsim KW: Quality control of Cordyceps sinensis, a valued traditional Chinese medicine. J Pharm Biomed Anal 41: 1571-1584, 2006.

8. Firenzuoli F, Gori L and Lombardo G: The medicinal mushroom Agaricus blazei Murrill: review of literature and pharmacotoxicological problems. Evid Based Complement Alternat Med 5: 3-15, 2008.

9. Schmidt BM, Ribnicky DM, Lipsky PE and Raskin I: Revisiting the ancient concept of botanical therapeutics. Nat Chem Biol 3: 360-366, 2007.
10. Zhao S, Ye G, Fu G, et al: Ganoderma lucidum exerts anti-tumor effects on ovarian cancer cells and enhances their sensitivity to cisplatin. Int J Oncol 38: 1319-1327, 2011.

11. Chihara G, Maeda Y, Hamuro J, Sasaki T and Fukuoka F: Inhibition of mouse sarcoma 180 by polysaccharides from Lentinus edodes (Berk.). Nature 222: 687-688, 1969.

12. Campling BG, Pym J, Galbraith PR and Cole SP: Use of the MTT assay for rapid determination of chemosensitivity of human leukemic blast cells. Leuk Res 12: 823-831, 1988.

13. Hahne JC, Honig A, Meyer SR, et al: Downregulation of AKT reverses platinum resistance of human ovarian cancers in vitro. Oncol Rep 28: 2023-2028, 2012.

14. Traganos F and Darzynkiewicz Z: Lysosomal proton pump activity: supravital cell staining with acridine orange differentiates leukocyte subpopulations. Methods Cell Biol 41: 185-194, 1994.

15. Jin X, Gossett DR, Wang S, et al: Inhibition of AKT survival pathway by a small molecule inhibitor in human endometrial cancer cells. Br J Cancer 91: 1808-1812, 2004.

16. Block M, Fister S, Emons G, et al: Antiproliferative effects of antiestrogens and inhibitors of growth factor receptor signaling on endometrial cancer cells. Anticancer Res 30: 2025-2031, 2010.

17. Tsujimoto Y and Shimizu S: Another way to die: autophagic programmed cell death. Cell Death Differ 12 (Suppl 2): 1528-1534, 2005.

18. González-Polo RA, Boya P, Pauleau AL, et al: The apoptosis/ autophagy paradox: autophagic vacuolization before apoptotic death. J Cell Sci 118: 3091-3102, 2005.

19. Ouyang L, Shi Z, Zhao S, et al: Programmed cell death pathways in cancer: a review of apoptosis, autophagy and programmed necrosis. Cell Prolif 45: 487-488, 2012.

20. Amaral C, Borges M, Melo S, et al: Apoptosis and autophagy in breast cancer cells following exemestane treatment. PLoS One 7: e42398, 2012.

21. Jiang J, Slivova V, Harvey K, Valachovicova T and Sliva D: Ganoderma lucidum suppresses growth of breast cancer cells through the inhibition of Akt/NF- $\mathrm{KB}$ signaling. Nutr Cancer 49: 209-216, 2004.

22. Jin CY, Kim GJ and Choi YH: Induction of apoptosis by aqueous extract of Cordyceps militaris through activation of caspases and inactivation of Akt in human breast cancer MDA-MB-231 cells. J Microbiol Biotechnol 18: 1997-2003, 2008.

23. Jiang J and Sliva D: Novel medicinal mushroom blend suppresses growth and invasiveness of human breast cancer cells. Int J Oncol 37: 1529-1536, 2010.

24. Calviño E, Manjón JL, Sancho P, et al: Ganoderma lucidum induced apoptosis in NB4 human leukemia cells: involvement of Akt and Erk. J Ethnopharmacol 128: 71-78, 2010.

25. Dotan N, Wasser SP and Mahajna J: Inhibition of the androgen receptor activity by Coprinus comatus substances. Nutr Cancer 63: 1316-1327, 2011.

26. Coutte L, Dreyer C, Sablin MP, Faivre S and Raymond E: PI3KAKT-mTOR pathway and cancer. Bull Cancer 99: 173-180, 2012 (In French).

27. Hahne JC, Meyer SR, Gambaryan S, et al: Immune escape of AKT overexpressing ovarian cancer cells. Int J Oncol 42: 1630-1635, 2013.

28. Honig A, Hahne JC, Meyer S, et al: PI3K inhibitor D-116883 is effective in in vitro models of ovarian cancer. Anticancer Res 32: 2035-2041, 2012.

29. Pant A, Lee II, Lu Z, et al: Inhibition of AKT with the orally active allosteric AKT inhibitor, MK-2206, sensitizes endometrial cancer cells to progestin. PLoS One 7: e41593, 2012.

30. Prasad R, Vaid M and Katiyar SK: Grape proanthocyanidin inhibit pancreatic cancer cell growth in vitro and in vivo through induction of apoptosis and by targeting the PI3K/Akt pathway. PLoS One 7: e43064, 2012.

31. Wang RC, Wei Y, An Z, et al: Akt-mediated regulation of autophagy and tumorigenesis through Beclin 1 phosphorylation. Science 338: 956-959, 2012.

32. Mizuno T: The extraction and development of antitumor-active polysaccharides from medicinal mushrooms in Japan (review). Int J Med Mushr 1: 9-30, 1999.

33. Wasser SP and Weis AL: Medicinal properties of substances occurring in higher Basidiomycetes mushrooms: current perspectives (review). Int J Med Mushr 1: 31-62, 1999.

34. Reshetnikov SV, Wasser SP and Tan KK: Higher basidiomycetes as a source of antitumor and immunostimulating polysaccharides (review). Int J Med Mushr 3: 361-394, 2001. 
35. Wasser SP: Medicinal mushrooms as a source of antitumor and immunomodulating polysaccharides. Appl Microbiol Biotechnol 60: 258-274, 2002.

36. Xie YZ, Li SZ, Yee A, et al: Ganoderma lucidum inhibits tumour cell proliferation and induces tumour cell death. Enzyme Microb Technol 40, 177-185, 2006.

37. Kiriakidis S, Högemeier O, Starcke S, et al: Novel tempeh (fermented soyabean) isoflavones inhibit in vivo angiogenesis in the chicken chorioallantoic membrane assay. Br J Nutr 93: 317-323, 2005 .
38. Wu WK, Coffelt $\mathrm{SB}$, Cho $\mathrm{CH}$, et al: The autophagic paradox in cancer therapy. Oncogene 31: 939-953, 2012.

39. Carew JS, Kelly KR and Nawrocki ST: Autophagy as a target for cancer therapy: new developments. Cancer Manag Res 4: 357-365, 2012. 